\title{
Cálculo do prazo de validade e da velocidade e distribuição da deterioraçáo de um produto panificado
}

\author{
Camila Santanna Oliveira ${ }^{1}$ \\ Miguel Afonso Sellitto ${ }^{2}$
}

\begin{abstract}
Resumo: O objetivo deste artigo é relatar uma pesquisa que investigou acerca do cálculo do prazo de validade e da velocidade de deterioração de um produto panificado. $\mathrm{O}$ método de pesquisa foi a experimentação de laboratório. Dez fatias de um produto panificado foram observadas por 130 dias em laboratório, em condiçóes similares às de venda, portanto antes do consumo. A deterioração começou no $14^{\circ}$ dia, que pode ser tomado como prazo de validade para o produto. Observou-se que no $30^{\circ}$ dia a distribuição da deterioração entre as fatias ajustou-se a uma distribuição normal. Observou-se ainda que a velocidade da deterioração ajustou-se a um modelo de regressão logística. Após 130 dias, todas as fatias deterioraram-se totalmente. Um grupo de dez fatias em condiçóes controladas de temperatura e umidade foi monitorado por 60 dias, sem qualquer deterioração observada.
\end{abstract}

Palavras-chave: Análise de sobrevivência, Tempo até a falha, Modelos probabilísticos, Modelo logístico.

\begin{abstract}
The purpose of this article is to report a research that has investigated about calculating the expiry date and the speed of deterioration of a baked product. The research method was the laboratory experimentation. Ten slices of a baked product were observed for 130 days in the laboratory, in similar conditions to the sale, prior to consumption. The deterioration began on the 14 th day, which can be taken as the validity for the product. It was observed that on the 30th day the distribution of decay among the slices fits to a normal distribution. It was also observed that the speed of deterioration fits to a logistic regression model. After 130 days, all the slices completely deteriorated. A group of ten slices under controlled conditions of temperature and humidity was monitored by 60 days, without any observed deterioration.
\end{abstract}

Keywords: survival analysis, time until failure, Probabilistic Models, logistic model.

Universidade do Vale do Rio dos Sinos - UNISINOS (milasantanna@hotmail.com)

2 Universidade do Vale do Rio dos Sinos - UNISINOS (sellitto@unisinos.br) 


\section{INTRODUÇÃO}

O tempo de vida ou tempo até a falha é o tempo de sobrevivência de um item, ou seja, o tempo decorrido desde a sua produção até a sua perda, falha ou inutilidade para uso. Este tempo é estudado pela análise de sobrevivência ou confiabilidade. A análise de sobrevivência estuda indivíduos (os itens que são observados em um experimento), com os quais ocorre, depois de algum tempo, um evento definido, a perda ou falha. É caracterizada por um conjunto de técnicas e modelos estatísticos usados na análise de situaçóes em que a variável de interesse é o tempo de sobrevivência. Este consiste no tempo decorrido desde a entrada de um indivíduo no estudo até a ocorrência do evento de interesse (falha) ou de uma censura. A censura ocorre quando, por alguma razáo, o acompanhamento do indivíduo é interrompido, seja porque a falha ocorreu por motivo diferente do estudado, ou porque o mesmo foi modificado e excluído da amostra, ou, ainda, porque se encerrou o tempo de observação (Colosimo e Giolo, 2006).

Para a análise de sobrevivência, é necessário compreender o conceito de confiabilidade, que é a capacidade de uma unidade observada satisfazer as exigências necessárias para a finalidade de uso, nos limites especificados para manter suas propriedades por um determinado tempo. Essa capacidade é descrita quantitativamente pelas funções de confiabilidade, que descrevem o resultado dos efeitos dos modos de falhas que podem ser observados durante o uso do material (Lewis, 1996).

Um modo de falha é o efeito observado causador de uma falha em um item. Como os itens são desenvolvidos para realizar uma ou mais funções, um modo de falha é definido como a não-realização de uma destas funçóes. A maioria dos itens apresenta múltiplos modos de falha. $\mathrm{O}$ tempo até a falha designa o período desde que a unidade entrou em operação até o momento da falha e é uma grandeza aleatória (Hoyland e Rausand, 1994).

É importante no estudo da análise de sobrevivência definir o que é perda ou falha. Perdas ou falhas geralmente são associadas a uma variável aleatória cujo comportamento pode ser descrito por um modelo probabilístico de engenharia (Hahn e Shapiro, 1967; Sidall, 1972).

São exemplos de variáveis aleatórias modeláveis o tempo até a falha de um componente in- dustrial, o tempo até a necessidade de um reparo, o tempo até o falecimento de um paciente com certo diagnóstico, etc. Neste artigo, o evento de falha foi a declaração de que um produto alimentício perdeu sua validade para comercialização e consumo e o tempo estudado foi o tempo decorrido desde a fabricação do produto até a sua inutilização para consumo humano.

O objetivo deste artigo foi descrever uma pesquisa experimental em que se determinou o prazo de validade, a velocidade de deterioração e a sua distribuiçáo em um produto panificado. $\mathrm{O}$ prazo de validade é obtido por análise de confiabilidade (ou sobrevivência), a velocidade de deterioração por análise de regressão e a sua distribuição por ajuste dos dados a modelos probabilísticos. $\mathrm{O}$ método de pesquisa foi o experimento de laboratório.

O restante do artigo está organizado em: revisão bibliográfica sobre a função sobrevivência, alguns dos modelos probabilísticos mais presentes na literatura e análise de regressão; descrição dos procedimentos experimentais e resultados obtidos; e discussão dos resultados e da pesquisa. A pesquisa foi inteiramente financiada pelo CNPq.

\section{FUNÇÓES DE SOBREVIVÊNCIA E PRINCIPAIS DISTRIBUIÇÓES}

A função sobrevivência (survival function) $S(t)$ é a probabilidade de não se observar falha até o tempo $t$. Em outras palavras, é a probabilidade de um item durar mais do que $t$ períodos de tempo (Colosimo e Giolo, 2006). É topologicamente equivalente à funçáo de confiabilidade (reliability funcion), a probabilidade de um item operar satisfatoriamente até o tempo $t$ (Elsayed, 1996). Para o seu cálculo, usa-se a Eq. 1.

$$
R(t)=\frac{n_{s}(t)}{n_{s}(t)+n_{f}(t)}=\frac{n_{s}(t)}{n_{0}}
$$

na qual:

$n_{0}=$ número total de itens testados.

$n_{f}(t)=$ número de itens que falharam durante o teste até o instante t.

$N_{s}(t)=$ número de itens que não falharam durante $\mathrm{o}$ teste até o instante t.

$n_{0}=n_{f}(t)+N_{s}(t)$ 
Considerando-se a Eq. 2, a função de confiabilidade $R(t)$ é o complemento da função acumulada de falha $F(t)$, como mostrado na Eq. 3 (Nelson, 1982).

$$
\begin{aligned}
& F(t)=\frac{n_{f}(t)}{n_{0}} \\
& R(t)=1-F(t)=P(T>1)
\end{aligned}
$$

na qual:

$T=$ tempo de falha

A função de risco (bazard funcion) é um indicador da propensão à falha após um tempo ter transcorrido sem falha. Relaciona a cada duração $t$ a probabilidade da falha ocorrer num instante de tempo infinitesimalmente sucessor a $t$. É, portanto, uma probabilidade condicional, pois o evento de falha é condicionado ao fato de não ter havido falha até aquele instante. A função de taxa de risco é dada pela Eq. 4 (Nelson, 1982).

$$
h(t)=\lim _{\Delta t \rightarrow 0} \frac{p[t \leq T \leq t+\Delta t) / T \geq t]}{\Delta t}=\frac{f(t)}{S(t)}(\mathrm{Eq} .
$$

A função de risco de um intervalo de tempo também pode ser expressa pela Eq. 5 .

$$
h\left(t_{I}\right)=\frac{n_{f}\left(\Delta_{i}\right)}{n_{s}\left(t_{i}-1\right) * \Delta t_{i}}
$$

na qual:

$h\left(t_{I}\right)=$ função de risco de tempo $t_{i \text {. }}$

$n_{s}\left(t_{i}-1\right)=$ número de falhas no intervalo $\Delta_{t i}$

$n_{s}\left(t_{i}-1\right)=$ número de sobreviventes no início do intervalo $t_{i}$.

$\Delta t_{i}=$ intervalo de tempo $\left(t_{i}-1, t_{i}\right)$.

A função de confiabilidade $R(t)$ e a função de risco $h(t)$ se relacionam pela Eq. 6 .

$$
R(t)=\exp \left(-\int_{0}^{t} h(\xi) d \xi\right)
$$

A seguir apresentam-se algumas distribuições para o tempo até a falha que são úteis em confiabilidade.

\subsection{MODELO EXPONENCIAL}

A distribuição exponencial é útil em fenômenos sem memória. Nestes, a chance condicio- nal de falha no intervalo de tempo de comprimento estipulado é a mesma, independente do tempo que o item venha a ser testado. A função de risco $\lambda$, entáo, é constante. Se $T$ tem distribuição exponencial com parâmetro $\lambda>0$, a funçáo sobrevivência ou confiabilidade é dada pela Eq. 7 .

$$
R(t)=e^{-\lambda t} \quad(\text { Eq. 7) }
$$

Outras características de interesse são as médias, a variância e os percentis. $\mathrm{O}$ percentil $100 p \%$ corresponde ao tempo em que $100 p \%$ dos produtos falharem, o p-ésimo quantil é $\alpha \log (1-p)$. A média da distribuição exponencial é $\lambda$ e a variância é $\lambda^{2}$. Os percentis são importantes quando se quer informaçóes a respeito de falhas prematuras (Souza, 2001).

O modelo exponencial é um caso especial do modelo de Weibull.

\subsection{MODELO WEIBULL}

Esta distribuição representa uma generalização da distribuição exponencial e é bastante utilizada no ajuste de dados de confiabilidade nas diversas áreas do conhecimento, entre elas a medicina e engenharia. $\mathrm{Na}$ engenharia, a distribuição Weibull é a principal função de confiabilidade, sendo utilizada para modelar a distribuição da vida útil e taxa de risco em produtos industriais do tipo serial, nos quais o primeiro componente que falha causa a falha do produto. Sua taxa de falha é monótona, ou seja, é decrescente, constante ou crescente, conforme o parâmetro de forma, sendo útil para modelar tempos de falha em equipamentos. Se $T$ tem distribuição de Weibull com parâmetros $\alpha>0$ e $\beta>0$, a função de confiabilidade é dada pela Eq. 8 (Lawless, 1982)

$$
R(t)=e^{-\left(\frac{t}{\alpha}\right)^{\beta}}
$$

Os parâmetros de forma $\beta$ e o de escala $\alpha$ são positivos. $O$ parâmetro $\alpha$ tem a mesma unidade de $t$ e $\beta$ é adimensional. Se $\beta=1$, a distribuição recai na exponencial. A função de risco $h(t)$ é dada pela Eq. 9 e é estritamente crescente para $\beta>1$; estritamente decrescente para $\beta<1$; e é constante para $\beta=1$ (exponencial). 


$$
h(t)=\frac{\beta}{\alpha} \cdot\left(\frac{t}{\alpha}\right)^{\beta-1}
$$

Segundo Silva (2002) e Collet (1994), o modelo de Weibull é tâo importante para a análise de confiabilidade quanto a distribuição normal o é para os modelos lineares e de gestão da qualidade.

\subsection{MODELO NORMAL}

A distribuição normal é a distribuição mais comumente utilizada em estatística e também uma das mais importantes das distribuições contínuas. Esta distribuição possui forma de sino (unimodal), onde o seu valor de máxima frequência (moda) coincide com o valor da média e da mediana. A média $(\mu)$ é o centro da curva e o desvio padrão $(\sigma)$ refere-se ao espalhamento da curva. A distribuição normal é simétrica em torno da média. A equação da curva normal é dada pela Eq. 10 (Mood e Graybill, 1974).

$$
f(x)=\frac{1}{\sqrt{2 \varpi}} * e^{\frac{(x-\mu)^{2}}{2 \sigma^{2}}}
$$

\subsection{DISTRIBUIÇÃO LOGNORMAL}

A lognormal é útil para tempos até a falha de semicondutores e de grupos de mecanismos que operam em conjunto. Outros exemplos são reaçôes químicas, tais como corrosão, movimento de materiais devido a difusão ou migração ou propagação de fraturas. É utilizada também para prognósticos de confiabilidade em testes de vida acelerada. É a distribuição mais indicada para se fazerem extrapolaçóes para valores bem abaixo dos menores valores obtidos. Por exemplo, se o menor ponto é $1 \%$ e necessita-se determinar porcentagens de $0,01 \%$. Assim como o limite da soma de distribuiçóes independentes tende a uma normal, o limite da multiplicaçáo tende para a lognormal. Sua função de densidade de probabilidade (PDF) é expressa pela Eq. 11 (Mood e Graybill, 1974).

$f(x)=\frac{1}{\sigma \sqrt{2 \pi}} e^{-\left(\frac{1}{2 \sigma^{2}}\right)}\left(\mathrm{h} t-\mathrm{h} T_{\mathrm{B}}\right)^{2}$ na qual:

$\mu=$ média do logaritmo dos dados.

$\sigma=$ desvio padrão do logaritmo dos dados.

$T_{50}=\mathrm{e}^{\mu}$

\subsection{MODELO GAMMA}

O modelo Gamma é adequado a sistemas em paralelo, ou seja, a falha ocorre quando o último de $n$ indivíduos falha. Uma relação existente entre a distribuição gama e a exponencial é o fato de a soma de exponenciais independentes e identicamente distribuídas possuir distribuição gamma. A função densidade de probabilidade é dada pela Eq. 12.

$$
f(t)=\left(\frac{t^{k-1}}{\alpha^{k} \Gamma(k)}\right) \cdot e^{-\left(\frac{t}{\alpha}\right)}
$$

A função de risco é monótona crescente para $k>1$; constante para $k=1$; e monótona decrescente para $0<k<1$ (Lewis, 1994).

\section{AJUSTE DE CURVAS POR REGRESSÁO}

Quando existe uma relação não aleatória entre variáveis e deseja-se expressar esta relação sob forma de uma equação, lança-se mão de regressóes matemáticas (Bussab, 1988).

A análise de regressão objetiva projetar ou interpolar valores em uma série de dados. Se houver uma teoria que justifique uma relação de causa e efeito, pode-se extrapolar, ou seja, sair dos domínios das variáveis. Do contrário, é mais prudente permanecer nos domínios das variáveis explicados pela regressão, limitando-se a interpolações entre os dados. Se a relação entre as variáveis for expressa por polinômio em $x^{n}, n$ inteiro, é chamada linear. A não-linearidade surge, por exemplo, quando a variável $x$ passa ao expoente, como em $y=e^{x}$.

Caso haja uma teoria apriorística, deve-se adotá-la na construçáo do modelo. Do contrário devem-se tentar alternativas e compará-las, segundo um critério. $\mathrm{Na}$ prática, o problema consiste em estimar os valores dos parâmetros da equação e entâo projetar prediçốes de valores intermediários ou além dos domínios das variáveis. No Quadro 1 , surgem alguns tipos de curvas de ajustamento e suas equaçóes (Bussab, 1988). 
Quadro 1: Curvas de ajustamento de dados

\begin{tabular}{|l|c|}
\hline \multicolumn{1}{|c|}{ Expressáo matemática } & descriçáo \\
\hline$Y=a_{0}+a_{1} x$ & $\begin{array}{c}\text { linha reta ou } \\
\text { linear }\end{array}$ \\
\hline$Y=a_{0}+a_{1} x+a_{2} x^{2}+\ldots+a_{n} x^{n}$ & $\begin{array}{c}\text { Polinômio de } \\
\text { enésimo grau }\end{array}$ \\
\hline$Y=a_{0}+a_{1} x^{22}$ & $\begin{array}{c}\text { hiperbólica ou } \\
\text { potência }\end{array}$ \\
\hline$Y=a b^{X}$ & exponencial \\
\hline$Y=\log a+b \log x$ & logarítmica \\
\hline
\end{tabular}

fonte: Bussab, 1988

É possível avaliar a qualidade da regressão pelo coeficiente de determinação $R^{2}$, calculado pela equaçáo 13 , variando de 0 a 1: quanto mais próximo de 1, melhor a regressão (Spiegel, 1978). $\mathrm{O} R^{2}$ não é o único parâmetro de avaliação de regressóes, mas é suficiente para este artigo.

$$
R^{2}=\frac{\sum(\hat{y}-\bar{y})^{2}}{\sum(y-\bar{y})^{2}}
$$

na qual:

$\hat{y}=$ estimativa pela regressão.

$y=$ observação.

$\bar{y}=$ média das observaçôes.

Para calcular os coeficientes da equação de regressão, um método é o dos mínimos quadrados, cujo objetivo é encontrar constantes tais que minimizem a soma dos erros quadráticos. Calculam-se os erros quadráticos médios, derivam-se as expressóes, igualam-se as mesmas a zero, chegando-se a um sistema de $n$ equaçóes normais a $n$ incógnitas. A solução oferece os coeficientes. As equaçôes normais para diversas regressôes podem ser encontrada em Spiegel (1978).

Outros métodos incluem aproximaçóes iterativas e são úteis quando o modelo é não-linear.

Os principais métodos têm formatos semelhantes: (i) estabelecem-se valores iniciais para as variáveis $x$; (ii) gera-se a curva definida pelas variáveis iniciais e calcula-se a soma quadrática de resíduos; (iii) segundo um dado algoritmo capaz de diminuir a soma quadrática, modificam-se as variáveis; e (iv) aplica-se recursivamente o algoritmo até que duas sucessivas aplicações não apresentem melhoria significativa. Pode ser desejável variar as estimativas iniciais e verificar o resultado e a velocidade de convergência (Draper e Smith, 1981).

Diversos métodos surgem na literatura. O método de Gauss-Newton ou da linearização expande o modelo náo-linear em uma série de Taylor, aproximando-o por um modelo linear. Estimam-se entáo os parâmetros $x_{i}$ pelo método dos mínimos quadrados em iteraçôes, até que um critério de término seja atingido. Há ainda o método da descida mais íngreme e o método de Levenberg-Marquardt (LM), que combina os outros dois. Se está longe da convergência, LM comporta-se como descida mais íngreme, se está próximo, como Gauss-Newton. Já o método GRC (generalized reduced gradient) melhora uma solução inicial, explorando várias direçôes, até que um critério encerre a exploração. Diferentes métodos podem chegar a resultados levemente diferentes (Draper e Smith, 1981).

\section{A PESQUISA}

A questão de pesquisa foi: como determinar um prazo de validade para a comercialização e consumo humano de um produto panificado? Uma questáo secundária foi: dado que iniciou a deterioração, como descrever sua velocidade e distribuição? O objetivo de pesquisa foi calcular por experimentação o prazo de validade, a velocidade de deterioração e sua distribuição para um produto panificado. Os objetivos intermediários foram: definição do produto; definição do método de experimentaçáo; realizaçáo do experimento; e análise matemática dos dados. Os tempos até o início da deterioração foram analisados por modelos de confiabilidade (ou sobrevivência) e, dado que esta se iniciou em $t$, a velocidade de deterioração foi descrita por modelos de regressão oferecidos pelo software livre Curve Expert e sua distribuição ajustada pelo software Proconf 98.

O produto escolhido foi o pão de sanduíche do tipo light, da marca WWW. A amostra analisada foi de $500 \mathrm{~g}$ (dez fatias de um mesmo lote produzido). O lote era 27:02:07:* $\mathrm{M}$ e a validade de 08:03:07:TA.

As fatias foram embaladas individualmente, para evitar a contaminação cruzada. Foram montadas duas levas, que foram deixadas sob duas 
condiçōes diferentes de temperatura e umidade relativa do ar.

Na primeira leva, deixou-se o pão nas condiçóes ambientes, com temperatura média de $21^{\circ} \mathrm{C}$ e umidade relativa do ar náo controlada por cento e trinta dias, que foi o tempo até que todas as fatias tivessem se deteriorado por completo. Para esta leva, esperava-se repetir as condições encontradas durante a etapa de venda do produto, como ocorre nos pontos de comercializaçáo, ou seja, antes do consumo. Após trinta dias, a deterioração observada já foi suficiente para determinar o modelo de avanço, pois todas as fatias já haviam se deteriorado em grau significativo. A partir desta data, apenas observou-se o avanço. Mediu-se a deterioração visualmente, pelo percentual de área das fatias ocupada pelos micro-organismos característicos da degradação.

$\mathrm{Na}$ segunda leva, deixou-se o pão na temperatura média de $37^{\circ} \mathrm{C}$ e umidade relativa do ar em $90 \%$, em estufa própria para panificados, por sessenta dias. As amostras não se deterioraram até o $60^{\circ}$ dia, quando se encerrou o experimento.

$\mathrm{Na}$ primeira leva, com amostras em temperatura média de $21^{\circ} \mathrm{C}$ e umidade relativa do ar náo controlada, a deterioração iniciou no $14^{\circ} \mathrm{dia}$. Du- rante os treze dias iniciais, não houve deterioração. Este resultado surpreendeu os pesquisadores, pois se esperava que o tempo até o início da deterioraçáo fosse uma grandeza probabilística. No experimento, porém, a grandeza mostrou-se a mesma para todos os indivíduos sob teste. Para o lote, concluiu-se que a grandeza é constante. A partir do $14^{\circ} \mathrm{dia}$ começou o crescimento de micro-organismos em todas as fatias. Resta investigar se o método de experimentaçáo foi responsável pela inexistência de variabilidade na grandeza investigada.

$\mathrm{O}$ avanço da deterioração foi medido até o $30^{\circ}$ dia, o que foi suficiente para o desenvolvimento do modelo. A velocidade de deterioração mostrou alguma aleatoriedade, avançando de modo diferente nas diferentes fatias. A observaçáo prosseguiu até o $130^{\circ}$ dia.

Os resultados estão na Tabela 1 . Na tabela, a deterioração é apresentada dia a dia, fatia por fatia. Nas três últimas colunas, é apresentada a média $\mu$ de deterioração entre as fatias, o desvio-padrão $\sigma$ e o coeficiente de variação $(\mathrm{cv}=\sigma / \mu)$. Após o $24^{\circ}$ dia, a deterioração pareceu estabilizar-se no patamar de $8 \%$. Após o $35^{\circ}$ dia, a deterioração avançou linearmente, até atingir $100 \% \mathrm{em}$ todas as fatias no $130^{\circ}$ dia.

Tabela 1: Resultados do experimento

\begin{tabular}{|c|c|c|c|c|c|c|c|c|c|c|c|c|c|}
\hline & \multicolumn{10}{|c|}{ Deterioraçáo da fatia } & \multicolumn{3}{|c|}{ Parâmetros } \\
\hline Dia & $1^{\mathrm{a}}$ & $2^{\mathrm{a}}$ & $3^{\mathrm{a}}$ & $4^{\mathrm{a}}$ & $5^{\mathrm{a}}$ & $6^{\mathrm{a}}$ & $7^{\mathrm{a}}$ & $8^{\mathrm{a}}$ & $9^{\mathrm{a}}$ & $10^{\mathrm{a}}$ & $\mu$ & $\sigma$ & $\mathrm{Cv}$ \\
\hline $1^{\mathrm{o}}$ & $0 \%$ & $0 \%$ & $0 \%$ & $0 \%$ & $0 \%$ & $0 \%$ & $0 \%$ & $0 \%$ & $0 \%$ & $0 \%$ & $0 \%$ & 0 & \\
\hline $2^{\mathrm{o}}$ & $0 \%$ & $0 \%$ & $0 \%$ & $0 \%$ & $0 \%$ & $0 \%$ & $0 \%$ & $0 \%$ & $0 \%$ & $0 \%$ & $0 \%$ & 0 & \\
\hline $3^{\mathrm{o}}$ & $0 \%$ & $0 \%$ & $0 \%$ & $0 \%$ & $0 \%$ & $0 \%$ & $0 \%$ & $0 \%$ & $0 \%$ & $0 \%$ & $0 \%$ & 0 & \\
\hline $4^{\mathrm{o}}$ & $0 \%$ & $0 \%$ & $0 \%$ & $0 \%$ & $0 \%$ & $0 \%$ & $0 \%$ & $0 \%$ & $0 \%$ & $0 \%$ & $0 \%$ & 0 & \\
\hline $5^{\circ}$ & $0 \%$ & $0 \%$ & $0 \%$ & $0 \%$ & $0 \%$ & $0 \%$ & $0 \%$ & $0 \%$ & $0 \%$ & $0 \%$ & $0 \%$ & 0 & \\
\hline $6^{\circ}$ & $0 \%$ & $0 \%$ & $0 \%$ & $0 \%$ & $0 \%$ & $0 \%$ & $0 \%$ & $0 \%$ & $0 \%$ & $0 \%$ & $0 \%$ & 0 & \\
\hline $7^{\mathrm{o}}$ & $0 \%$ & $0 \%$ & $0 \%$ & $0 \%$ & $0 \%$ & $0 \%$ & $0 \%$ & $0 \%$ & $0 \%$ & $0 \%$ & $0 \%$ & 0 & \\
\hline $8^{\circ}$ & $0 \%$ & $0 \%$ & $0 \%$ & $0 \%$ & $0 \%$ & $0 \%$ & $0 \%$ & $0 \%$ & $0 \%$ & $0 \%$ & $0 \%$ & 0 & \\
\hline $9^{\circ}$ & $0 \%$ & $0 \%$ & $0 \%$ & $0 \%$ & $0 \%$ & $0 \%$ & $0 \%$ & $0 \%$ & $0 \%$ & $0 \%$ & $0 \%$ & 0 & \\
\hline $10^{\circ}$ & $0 \%$ & $0 \%$ & $0 \%$ & $0 \%$ & $0 \%$ & $0 \%$ & $0 \%$ & $0 \%$ & $0 \%$ & $0 \%$ & $0 \%$ & 0 & \\
\hline $11^{\circ}$ & $0 \%$ & $0 \%$ & $0 \%$ & $0 \%$ & $0 \%$ & $0 \%$ & $0 \%$ & $0 \%$ & $0 \%$ & $0 \%$ & $0 \%$ & 0 & \\
\hline $12^{\circ}$ & $0 \%$ & $0 \%$ & $0 \%$ & $0 \%$ & $0 \%$ & $0 \%$ & $0 \%$ & $0 \%$ & $0 \%$ & $0 \%$ & $0 \%$ & 0 & \\
\hline $13^{\circ}$ & $0 \%$ & $0 \%$ & $0 \%$ & $0 \%$ & $0 \%$ & $0 \%$ & $0 \%$ & $0 \%$ & $0 \%$ & $0 \%$ & $0 \%$ & 0 & \\
\hline $14^{\circ}$ & $2 \%$ & $2 \%$ & $2 \%$ & $2 \%$ & $2 \%$ & $2 \%$ & $2 \%$ & $2 \%$ & $2 \%$ & $2 \%$ & $2,0 \%$ & 0 & 0 \\
\hline
\end{tabular}




\begin{tabular}{|c|c|c|c|c|c|c|c|c|c|c|c|c|c|}
\hline & \multicolumn{10}{|c|}{ Deterioraçáo da fatia } & \multicolumn{3}{|c|}{ Parâmetros } \\
\hline $15^{\circ}$ & $3 \%$ & $3 \%$ & $3 \%$ & $3 \%$ & $3 \%$ & $3 \%$ & $3 \%$ & $3 \%$ & $3 \%$ & $3 \%$ & $3,0 \%$ & 0 & 0 \\
\hline $16^{\circ}$ & $3 \%$ & $3 \%$ & $3 \%$ & $3 \%$ & $3 \%$ & $3 \%$ & $3 \%$ & $3 \%$ & $3 \%$ & $3 \%$ & $3,0 \%$ & 0 & 0 \\
\hline $17^{\circ}$ & $4 \%$ & $4 \%$ & $4 \%$ & $4 \%$ & $4 \%$ & $4 \%$ & $4 \%$ & $4 \%$ & $4 \%$ & $4 \%$ & $4,0 \%$ & 0 & 0 \\
\hline $18^{\circ}$ & $4 \%$ & $4 \%$ & $5 \%$ & $4 \%$ & $4 \%$ & $4 \%$ & $5 \%$ & $4 \%$ & $4 \%$ & $5 \%$ & $4,3 \%$ & 0,005 & $11,23 \%$ \\
\hline $19^{\circ}$ & $5 \%$ & $5 \%$ & $6 \%$ & $5 \%$ & $5 \%$ & $5 \%$ & $6 \%$ & $5 \%$ & $5 \%$ & $6 \%$ & $5,3 \%$ & 0,005 & $9,11 \%$ \\
\hline $20^{\circ}$ & $6 \%$ & $5 \%$ & $7 \%$ & $5 \%$ & $5 \%$ & $6 \%$ & $7 \%$ & $6 \%$ & $6 \%$ & $7 \%$ & $6,0 \%$ & 0,008 & $13,61 \%$ \\
\hline $21^{\circ}$ & $6 \%$ & $6 \%$ & $7 \%$ & $6 \%$ & $6 \%$ & $6 \%$ & $7 \%$ & $6 \%$ & $7 \%$ & $7 \%$ & $6,4 \%$ & 0,005 & $8,07 \%$ \\
\hline $22^{\circ}$ & $6 \%$ & $6 \%$ & $7 \%$ & $6 \%$ & $7 \%$ & $7 \%$ & $7 \%$ & $6 \%$ & $8 \%$ & $7 \%$ & $6,7 \%$ & 0,007 & $10,07 \%$ \\
\hline $23^{\circ}$ & $7 \%$ & $7 \%$ & $7 \%$ & $6 \%$ & $7 \%$ & $7 \%$ & $7 \%$ & $7 \%$ & $8 \%$ & $8 \%$ & $7,1 \%$ & 0,006 & $8,00 \%$ \\
\hline $24^{\circ}$ & $7 \%$ & $7 \%$ & $8 \%$ & $6 \%$ & $7 \%$ & $8 \%$ & $8 \%$ & $8 \%$ & $8 \%$ & $8 \%$ & $7,5 \%$ & 0,007 & $9,43 \%$ \\
\hline $25^{\circ}$ & $7 \%$ & $7 \%$ & $8 \%$ & $7 \%$ & $7 \%$ & $8 \%$ & $8 \%$ & $8 \%$ & $9 \%$ & $9 \%$ & $7,8 \%$ & 0,008 & $10,11 \%$ \\
\hline $26^{\circ}$ & $8 \%$ & $7 \%$ & $8 \%$ & $7 \%$ & $7 \%$ & $8 \%$ & $8 \%$ & $8 \%$ & $10 \%$ & $9 \%$ & $8,0 \%$ & 0,009 & $11,79 \%$ \\
\hline $27^{\circ}$ & $8 \%$ & $7 \%$ & $8 \%$ & $7 \%$ & $7 \%$ & $8 \%$ & $8 \%$ & $8 \%$ & $10 \%$ & $9 \%$ & $8,0 \%$ & 0,009 & $11,79 \%$ \\
\hline $28^{\circ}$ & $8 \%$ & $7 \%$ & $8 \%$ & $7 \%$ & $7 \%$ & $8 \%$ & $8 \%$ & $8 \%$ & $10 \%$ & $9 \%$ & $8,0 \%$ & 0,009 & $11,79 \%$ \\
\hline $29^{\circ}$ & $8 \%$ & $7 \%$ & $8 \%$ & $7 \%$ & $7 \%$ & $8 \%$ & $8 \%$ & $8 \%$ & $10 \%$ & $9 \%$ & $8,0 \%$ & 0,009 & $11,79 \%$ \\
\hline $30^{\circ}$ & $8 \%$ & $7 \%$ & $8 \%$ & $7 \%$ & $7 \%$ & $8 \%$ & $8 \%$ & $8 \%$ & $10 \%$ & $9 \%$ & $8,0 \%$ & 0,009 & $11,79 \%$ \\
\hline
\end{tabular}

\section{DISCUSSÃO DOS RESULTADOS}

$\mathrm{Na}$ segunda leva, as amostras não deterioraram durante os sessenta dias da observação. Isto pode ter ocorrido por alguns fatores, entre eles, a embalagem escolhida pode ter deixado migrar umidade do páo para o ambiente, diminuindo assim a atividade de água na amostra. Ou, ainda, as matérias-primas utilizadas na produção deste pão estavam em tais condiçôes de conservação, que tornaram as fatias livres de contaminantes. Portanto, esta leva foi de menor interesse para o experimento.

$\mathrm{Na}$ primeira leva, as nas amostras que foram deixadas nas condiçóes ambientes, com temperatura média de $21^{\circ} \mathrm{C}$ e umidade relativa do ar não controlada, houve deterioração e foi possível construir o gráfico de avanço da deterioração média das fatias em função do tempo. $\mathrm{O}$ gráfico foi elaborado com o auxílio do programa Curve Expert (1.3), como mostra a Figura 1. A curva indicada pelo software como a de melhor aderência aos dados foi a do modelo logístico, informado na Tabela 2. O $R^{2}$ calculado, próximo a 1 , indica que o modelo é satisfatório.

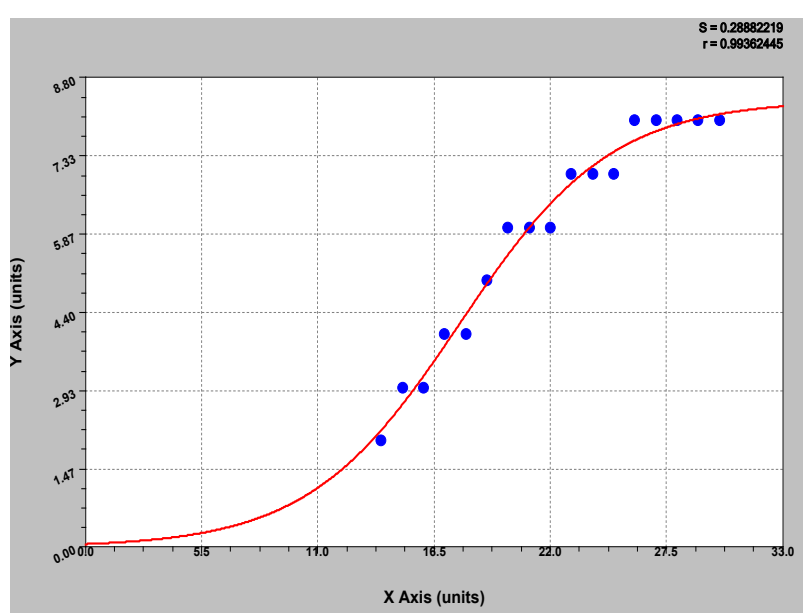

Figura 1: Velocidade de deterioração em função do tempo de atravessamento

Fonte: autores, com o uso do Curve Expert 1.3

Tabela 2: Modelo logístico

\begin{tabular}{|c|c|}
\hline Logistic Model & $y=a /\left[1+b \cdot \mathrm{e}^{-c x}\right]$ \\
\hline $\mathrm{a}=$ & 8.3776871 \\
\hline $\mathrm{b}=$ & 139.96314 \\
\hline $\mathrm{c}=$ & 0,27923776 \\
\hline Erro padrão $=$ & 0,2888222 \\
\hline$R^{2}=$ & 0,987 \\
\hline
\end{tabular}

Fonte: autores, com o uso do Curve Expert 1.3 
A variabilidade dos dados cresceu nos últimos dias observados, o que pode ser constatado pela evolução do $c v$, na Tabela 1 . Atingido o patamar de estabilidade, após o $30^{\circ}$ dia, a deterioração se distribuiu conforme o modelo normal. O teste foi feito com o auxílio do software ProConf 98. Quanto ao formato do crescimento, este é sintetizado na Figura 2, e é composto de uma fase exponencial e de uma fase de estabilidade.

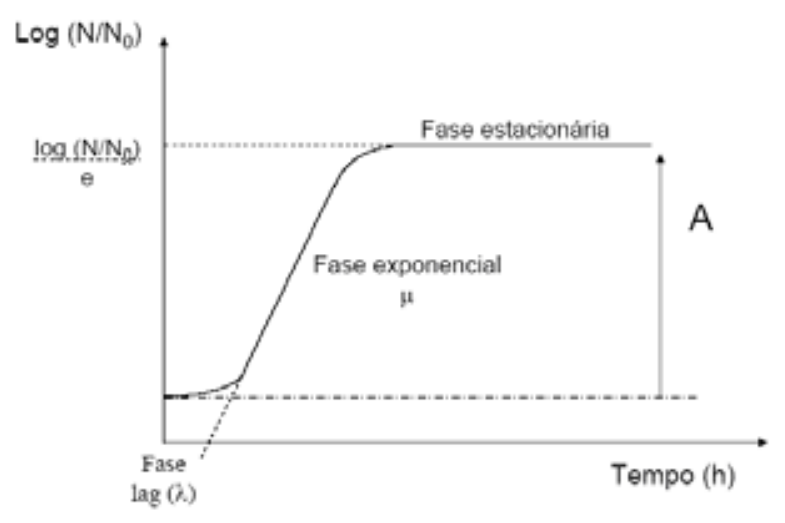

Figura 2: Curva de crescimento microbiano.

Sabe-se que os micro-organismos, para se desenvolverem, necessitam de água, alguma atividade baseada em água, uma fonte de energia, o açúcar, uma fonte de nitrogênio, os aminoácidos, e fontes de crescimento, as vitaminas. Também se sabe que os micro-organismos crescem, principalmente, em alimentos ácidos e com atividade de água mediana, tais como frutas, vegetais, cereais (principalmente úmidos) e queijos. Algumas espécies de bolores podem produzir micotoxinas, nocivas ao organismo humano por serem cumulativas. Esta é a principal razão para o cuidado de se produzir alimentos livres de contaminação microbiana.

Para que ocorra a deterioração microbiana de alimentos à base de cereais, farinhas, produtos de panificação, etc. são necessárias a presença de água livre (atividade de água) e contaminação por micro-organismos em geral. Quando a atividade de água é favorável, podem se desenvolver bacilos e bolores. $\mathrm{O}$ aumento de umidade que eleva a atividade de água pode ser introduzido no alimento, por exemplo, quando o mesmo é embalado ainda quente, propiciando a ocorrência da deterioração precoce.

Assim, pode-se explicar a demora na deterioração das amostras mantidas na condição 1 pelo uso de matérias-primas de boa qualidade na formulação do pão, por o processo de fabricação do pão ser livre de contaminantes, e pelo tratamento térmico eficiente, entre outros fatores.

Conclui-se que um prazo de validade aceitável para o produto estudado é de quatorze dias: os treze dias observados, mais um dia de distância entre a fabricação e a compra da amostra. Também conclui-se que o avanço da deterioração microbiana no produto segue o modelo logístico no tempo e a distribuição normal quanto às unidades do produto.

\section{CONCLUSÃO}

O objetivo deste trabalho foi calcular um prazo de validade e obter um modelo probabilístico para a velocidade de deterioraçáo de um produto panificado. $\mathrm{O}$ método de pesquisa empregado foi o experimento de laboratório.

Os dados obtidos foram analisados com o apoio de dois softwares, o Curve Expert 1.3 e o Proconf 98, perfazendo, respectivamente, a análise de regressão e o ajuste de modelos probabilísticos. A análise dos dados permitiu concluir que o tempo até o início da deterioração, que é a grandeza na qual se baseia o prazo de validade, nâo apresentou variabilidade, não sendo cabível o ajuste a modelos probabilísticos. A deterioração iniciou-se no $14^{\circ} \mathrm{dia}$, portanto a recomendação é que o prazo de validade seja fixado em quatorze dias, os treze dias observados, mais um dia até a compra da amostra.

Dado que a deterioraçáo iniciou, sua velocidade de avanço seguiu o modelo logístico. A distribuiçáo da deterioração nas fatias, em uma mesma data, seguiu o modelo normal.

Como continuidade de pesquisa, é recomendada a extensão do método experimental a outros tipos de produtos, tais como cárneos e laticínios e um experimento que investigue se a ausência de variabilidade no início da deterioração é devida ao método experimental empregado ou se é uma característica originada no processo de fabricação do produto.

\section{REFERÊNCIAS BIBLIOGRÁFICAS}

Bussab, W. (1988) Análise de variância e de regressão. São Paulo: Atual. 
Collet, D. (1994) Modelling Survival Data in Medical Research. London: Chapman and Hall.

Colosimo, E. (1995) Análise de Sobrevivência Aplicada. In: 46a Reunião Anual da Região Brasileira da Sociedade Internacional de Biometria (RBRAS) e 9o Simpósio de Estatística Aplicada e Experimentação Agronômica (SEAGRO). Piracicaba.

Colosimo, E., Giolo, S. (2006) Análise de sobrevivência aplicada. S. Paulo: Edgard Blücher.

Draper, N.; Smith, H. (1981) Applied Regression Analysis. N. York: John Wiley \& Sons.

Elsayed, E. (1996) Reliabillity engineering. Reading, MA: Addison Wesley Longman.

Hahn, G., Shapiro, S. (1967) Statistical Models in Engineering. NewYork: John Wilwy \& Sons.

Holand. A; Rausand. M.; (1994) System Reliability Theory: Models and Statistical Methods. New York: Wiley-Interscience.

Lawless, J. (1982) Statistical Models and Methods for Lifetime Data. New York: Wiley.
Lewis, E. (1996) Introduction to reliability engineering. New York: John Wiley \& Sons.

Mood, A., Graybill, F. (1974) Introduction to the theory of statistics. Tokyo: McGraw-Hill.

Nelson, W. Applied life data analysis. New York: John Wiley \& Sons, 1982. 634 p.

Silva, L. (2002) Estudo Comparativo entre o Modelo paramétrico Weibull e o Modelo Semi-Paramétrico de Cox. Natal: Monografia (Graduação em Estatística). Departamento de Estatística, Universidade Federal do Rio Grande do Norte.

Souza, E. (2001) Análise de Confiabilidade: Um estudo sobre o Tempo de Vida de Pneus. Natal: Monografia (Graduação em Estatística). Departamento de Estatística, Universidade Federal do Rio Grande do Norte.

Sidall, J. (1972) Analytical Decision-Making in Engineering Design. New York: Prentice-Hall.

Spiegel, M. (1978) Probabilidade e estatística, S. Paulo: McGraw-Hill. 